\title{
Concomitant intensive chemoradiotherapy induction in non-metastatic inflammatory breast cancer: long-term follow-up
}

\author{
D Genet', C Lejeune', P Bonnier², Y Aubard', L Venat-Bouvet', DJ Adjadj², J Martin', JL Labourey', \\ $A$ Benyoub ${ }^{2}, P$ Clavère', V Lebrun-Ly', $P$ Juin ${ }^{2}, L^{\prime}$ Piana $^{2}$ and $N$ Tubiana-Mathieu, \\ 'Department of medical oncology, CHU Dupuytren, Limoges, France; ${ }^{2}$ Department of medical oncology, CHU de la Conception, Marseille, France
}

The aim of this study was to evaluate with a long follow-up the efficacy of concomitant chemoradiotherapy in non-metastatic inflammatory breast cancer (IBC) and to evaluate the breast conservation rate. Between 1990 and 2000, 66 non-metastatic patients with IBC were treated with chemotherapy and concomitant irradiation. The induction chemotherapy consisted of epirubicine, cyclophosphamide and vindesine, in association with split-course bi-fractionated irradiation to a total dose of 65 Gy with concomitant cisplatin and 5-fluorouracil. Maintenance chemotherapy consisted of high-dose methotrexate and six cycles of epirubicine, cyclophosphamide and fluorouracil. Hormonal treatment was given if indicated. Mastectomy was not systemic. Among 65 evaluable patients, 57 (87.6\%) achieved a complete clinical response and had a breast conservation. Only six loco regional relapses were noted in six patients with a delay of 20 months and with concomitant metastatic dissemination in four cases. Median disease-free survival (DFS) was 28 months. Median overall survival (OS) was 63 months and median follow-up was 55.5 months. Induction chemotherapy and concomitant irradiation is feasible in patients with IBC, permitting a breast conservation with a high rate of local control with an OS comparable to that of the best recent series.

British Journal of Cancer (2007) 97, 883-887. doi: 10.1038/sj.bjc.6603987 www.bjcancer.com

Published online 18 September 2007

c) 2007 Cancer Research UK

Keywords: inflammatory breast cancer; chemoradiotherapy; breast conservation

Inflammatory breast cancer (IBC) accounts for $1-6 \%$ of all invasive breast tumours (Chang et al, 1998). Its incidence has doubled over the last 20 years (Hance et al, 2005).

Despite advances in treatment with induction chemotherapy regimens, disease-free survival (DFS) and overall survival (OS) remain unsatisfactory with a $30-50 \% 5$-year survival in the largest studies (Anderson et al, 2003; Cristofanilli et al, 2003; Somlo et al, 2004; Lerebours et al, 2005). The role of surgery remains controversial even though it is often used in protocols (Schafer et al, 1987; De Boer et al, 2000). The achievement of pathologic response to induction chemotherapy is an excellent indicator of prolonged (DFS) and (OS) (Ueno et al, 1997). This is achieved in less than $30 \%$ of patients (Chevallier et al, 1993).

Based on these results, we conducted a protocol combining the most active drugs such as anthracycline with radiotherapy as the major local treatment as the initial treatment without mastectomy.

We report the long-term efficacy of this protocol in 66 patients with non-metastatic IBC.

\section{MATERIALS AND METHODS}

Between 1990 and 2000, 66 consecutive patients with nonmetastatic IBC received treatment. All the patients were examined

\footnotetext{
*Correspondence: Professor N Tubiana-Mathieu;

E-mail: oncologie@chu-limoges.fr

Received 8 May 2007; revised 7 August 2007; accepted 14 August 2007;

published online 18 September 2007
}

by a multidisciplinary team and gave their written informed consent for participation. Eligibility included clinical and/or pathological criteria for IBC as defined by Haagensen (Haagensen, 1971). Non-metastatic IBC was defined by negative thoracoabdominal scan and bone scan.

The protocol was described previously (Tubiana-Mathieu et al, 2001) as shown in Figure 1: three cycles with a 28-day delay associated in day 1 , epirubicine (E) $\left(50 \mathrm{mg} \mathrm{m}^{-2}\right)$, cyclophosphamide $(\mathrm{C})\left(350 \mathrm{mg} \mathrm{m}^{-2}\right)$ and vindesine $(\mathrm{V})\left(2 \mathrm{mg} \mathrm{m}^{-2}\right)$, and CDDP $\left(10 \mathrm{mg} \mathrm{m}^{-2} \mathrm{day}^{-1}\right), 5$-FU $\left(500 \mathrm{mg} \mathrm{m}^{-2} \mathrm{day}^{-1}\right)$ in days $15-20$ concomitantly with bifractioned radiotherapy. After the first 24 patients, the doses of chemotherapy were increased as follows: $\mathrm{C}$ $500 \mathrm{mg} \mathrm{m}^{-2}$, E $75 \mathrm{mg} \mathrm{m}^{-2}$ and CDDP $20 \mathrm{mg} \mathrm{m}^{-2}$ days 15-20. Radiotherapy was performed as split course to a dose of $45 \mathrm{~Gy}$ with a twice daily schedule of $15 \mathrm{~Gy}$ for each course in 10 fractions for 5 days ( $1.5 \mathrm{~Gy}$ per fraction). The three series were performed from day 15 to 20 of each induction course concomitantly with CDDP and 5-FU. The volume included the whole breast by two tangential portals and supra clavicular, internal mammary chain, and eventually axillary lymph nodes by direct portal (axillary lymph nodes were not radiated after axillary dissection).

All volumes were treated by a ${ }^{60} \mathrm{Co}$ or six megavolt photon beam except the internal mammary chain, which was treated by a mixed $50-60 \%$ beam with photon and electron beam of adequate energy.

A boost irradiation course was performed 8 days after the 3 rd series. Twenty-four to $26 \mathrm{~Gy}$ (calculated as ICRU criteria) in $12-13$ daily fractions schedule over 16-17 days were administered to the entire breast, including the skin, but not the rib cage. This boost course was given without chemotherapy. 



Figure I Induction treatment protocol plan. Three Cycles with 28 days delay.

Methylprednisolone $\left(60 \mathrm{mg} \mathrm{D}^{-1}\right.$ po) and Warfarin $\left(1 \mathrm{mg} \mathrm{D}^{-1} \mathrm{po}\right)$ were given for the first 3 months because $30 \%$ of the initial 20 patients developed upper limb thrombophlebitis. All cycles were supported by granulocyte colony stimulating factor given from day 3 to 9 of each cycle.

After the radio chemotherapy programme, patients received three cycles of high-dose methotrexate (MTX) $\left(1.5 \mathrm{~g} \mathrm{~m}^{-2}\right)$ every 15 days and six cycles of FEC $50\left(5-\mathrm{FU} 500 \mathrm{mg} \mathrm{m}^{-2}, \mathrm{E} 50 \mathrm{mg} \mathrm{m}^{-2}\right.$ and C $500 \mathrm{mg} \mathrm{m}^{-2}$ ) every 21 days as maintenance chemotherapy.

Patients with ER and/or PR positive breast cancer received hormonotherapy: tamoxifen $20 \mathrm{mg} \mathrm{day}^{-1}$ for 5 years in pre- and postmenopausal patients after the end of chemotherapy and with analogue LH-RH in premenopausal patients.

The clinical response was evaluated after each cycle of the induction treatment. Multiple breast biopsies were performed after the induction from 1994.

Surgery (mastectomy or quadrantectomy) was performed only in case of stable disease or partial clinical or pathologic response.

After completion of the treatment, patients underwent physical examination at least once every 4 months for the first 2 years and every 6 months for 5 years. A yearly mammogram was performed, with bone scans and chest X-rays only according to symptoms.

\section{STATISTICAL METHODS}

The primary end point of this study was clinical tumour response defined in 1990 as follows: a complete response (CR) was defined as the complete disappearance of all clinical evidence of disease; partial response (PR) corresponded to a greater than $50 \%$ decrease in tumour area; no change (NC) was less change than partial regression without progressive disease; and progressive disease (PD) corresponded to $25 \%$ or more increase in tumour area or increased nodal stage or both.

Pathological response was assessed by six true-cut biopsies performed after chemoradiotherapy. Pathological response was complete (pCR) with no residual tumour cell on all biopsies.

Overall survival and DFS were calculated from diagnosis to last contact for living patients. Follow-up was calculated with the same variables. Disease-free survival was defined as the time of any type of recurrence or death of any cause. The standard Kaplan Meier method was applied for survival analysis.

Toxicities of treatment were graded according to the WHO scale.

\section{RESULTS}

\section{Patients' characteristics}

Median age was 48 years (25-70). Thirty-four were premenopausal and 32 postmenopausal.

The initial biopsy of the breast and the skin performed in all patients found 53 ductal, 11 lobular and two undifferentiated carcinoma. Sixty-two had clinical IBC with dermal lymphatic involvement in 43 patients. Four had occult disease, all with dermal lymphatic involvement.
To limit the radiotherapy fields, an axillary surgery was performed in the first 45 patients at the same time of indwelling catheter placement. Nodes picking were performed in nine patients, with 1-5 nodes being removed, and all were involved. Thirty-six axillary dissections were performed, with a median of 15 nodes being removed $(8-32)$; $84 \%$ showed 7 or more involved nodes.

Hormone receptor status was known in 58 patients. Estrogen/ progesterone receptors were positive/positive in 19 patients, negative/negative in 28 , and positive/negative in 11 .

\section{Evaluation of response}

The clinical response was evaluated in 65 patients; one was not evaluated because of death from pulmonary embolism at day 15 .

Fifty-seven patients (87.6\%) achieved clinical CR, seven (11\%) a clinical PR, and one a progression disease on the skin.

CR was obtained after two courses in 13 patients (38\%) and after three courses in 44 patients $(62 \%)$.

Pathological response was assessed in 25 patients: 22 had a pathologic complete response (pCR).

Among the seven patients with a partial clinical response, mastectomy was performed in five patients: in two patients, viable cells were not found in the breast (these two patients had a long time survival of 51 and 80 months without relapse) and in three patients, tumour viable cells were found (two patients developed metastasis and one had no relapse after surgery). For the other two patients, mastectomy was not performed due to the dramatic evolution: one developed brain metastases at 5 months and died at 7 months and the other after tumorectomy developed pulmonary metastasis at 6 months and died at 16 months.

\section{Evaluation of survival}

The median follow-up of the 66 patients was 55.5 months (range $4-178)$ in all patients and 89 months $(28-178)$ in surviving patients.

Among the 66 patients, 37 deaths occurred. All but four were related to breast cancer (the four non-related deaths were pulmonary embolism at 3 months, acute lymphoblastic leukaemia at 13 months, heart failure at 28 months and cirrhosis at 75 months). Median OS was 63 months and median DFS was 28 months. The 5- and 10 -year OS were 50.3 and $38 \%$, respectively (Figures 2 and 3).

\section{Pattern of failure}

As shown in Table 1, six patients developed local recurrence after a median delay of 20 months (range 7-42); two recurrences were only local without any distant metastasis and were alive without other recurrence at 56 and 89 months, and for the other four patients, local recurrence was associated with metastatic evolution. Five patients presented contralateral disease, with a median delay of 26 months (range 6-50). Two of them were isolated and three in association with distant metastasis. Thirty-six showed a systemic 


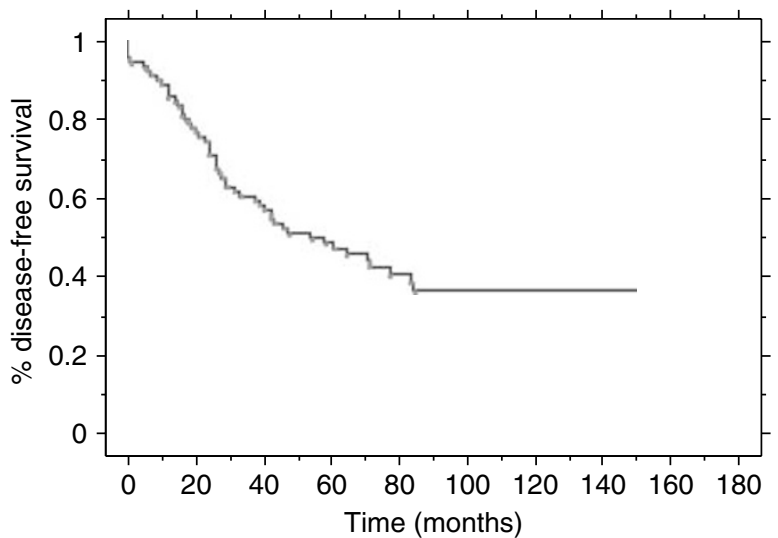

Figure 2 Disease-free survival for all patients.

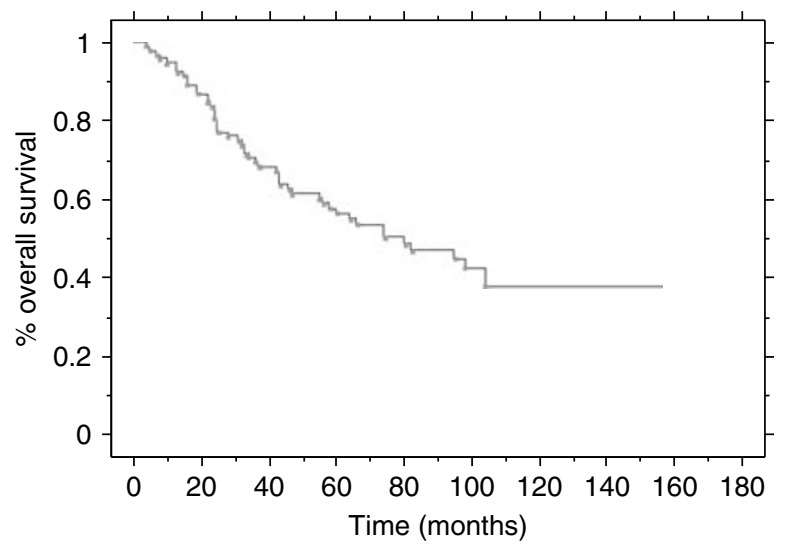

Figure 3 Overall Survival for all patients.

Table I Pattern of failure

\begin{tabular}{lcc}
\hline Pattern of failure & Number & Median delay in months (range) \\
\hline Local recurrence & 6 & $20(7-42)$ \\
Alone & 2 & \\
With systemic recurrence & 4 & $26(6-50)$ \\
Controlateral disease & 5 & \\
Alone & 2 & \\
With systemic recurrence & 3 & $19.5(1-84)$ \\
Systemic recurrence & 36 & \\
\hline
\end{tabular}

recurrence (seven patients had distant metastasis and local or contralateral recurrence), with a median delay of 19.5 months $(1-84)$. Sites of metastases were principally liver (19 patients), bone (11 patients), lung (6 patients) and brain (6 patients).

\section{Treatment toxicities}

As shown in Tables $2 \mathrm{~A}$ and $2 \mathrm{~B}$, the toxicity of chemotherapy during the induction phase was mainly haematologic with grade $3-4$ neutropaenia in 33 patients (50\%) and febrile neutropaenia in 8 patients (12\%) without septic death. Grade 3-4 thrombopaenia occurred in 16 patients $(24 \%)$ and grade $3-4$ anaemia occurred in three patients (5\%). During the maintenance phase, grade 3-4 neutropaenia occurred in 13 patients and grade 3-4 thrombopaenia occurred in 2 patients. Thrombosis occurred in eight patients (five cases occurred before the initiation of the treatment
Table 2A Induction haematological toxicity (worst toxicity for any course)

G III-IV (\%)

\begin{tabular}{lc}
\hline Anaemia & $3(5 \%)$ \\
Neutropaenia & $33(50 \%)$ \\
Febrile neutropaenia & $8(12 \%)$ \\
Thrombocytopaenia & $16(24 \%)$ \\
\hline
\end{tabular}

Table 2B Induction non-haematological toxicity

\begin{tabular}{lc}
\hline Nausea vomiting & $4(3 \mathrm{G}\|, \mathbf{I} \mathrm{G}\|)$ \\
Neuropathy & $3(\mathrm{G} \|)$ \\
Phlebitis & 8 \\
Mucositis & $2(\mathrm{G} \| 1)$ \\
Rib fracture & 6 \\
Serum transaminase & $7(3 \mathrm{G} \|, 4 \mathrm{G}$ II) \\
\hline
\end{tabular}

with warfarin; despite this preventive measure, two further cases occurred, one of which had the pulmonary embolism previously cited while receiving warfarin and then heparin). Other toxicities: two patients developed grade 3 serum transaminase elevation, two had grade 2 paraesthesias induced by cisplatin and two had grade 3 mucositis.

Radiotherapy was well tolerated during induction phase. One skin toxicity in an obese patient was noted with a local abscess 10 months after the end of treatment. In six patients, the principal side effect was rib fractures that occurred with a median delay of 12 months after the treatment.

One cardiac injury-related death was noted 31 months after diagnosis. The patient had been treated in childhood for an acute lymphoblastic leukaemia but had a normal cardiac function before breast cancer treatment.

\section{Feasibility}

Fifty-five (83\%) patients received $100 \%$ of the induction doses, 47 (71\%) patients received $100 \%$ of the MTX dose, and $47(71 \%)$ patients received $100 \%$ of the maintenance FEC 50 dose. Among the 41 patients who received higher doses of chemotherapy, 35 (85\%), $27(65 \%)$ and $29(70 \%)$, respectively, received $100 \%$ of induction, MTX and maintenance doses. The dose of anthracycline was never decreased. All patients received the protocol radiation dose. The median duration of radiation therapy was 81 days (range $60-98$ ), compared with 70 days as initially planned. The median duration of treatment was 9 months (range 2-13).

\section{DISCUSSION}

This study, with a long follow-up of 55.5 months, had demonstrated the role of intensive initial chemoradiotherapy to improve local control in IBC and to avoid mastectomy with a long survival. Our population included very homogeneous patients.

The best outcome for these patients classically uses a multimodality therapy, including neoadjuvant chemotherapy, surgery, adjuvant chemotherapy, radiotherapy and endocrine therapy in case of positive receptors.

Radiotherapy was the mainstay of care for IBC. In a trial published in 1987, Schafer et al (1987) demonstrated that patients treated with neoadjuvant chemotherapy, surgery and adjuvant chemotherapy but without radiotherapy developed local recurrences 8-17 months after mastectomy. In the present trial, we used an early split course twice daily radiation therapy in combination with chemotherapy. The treatment with a bifractionated irradiation technique, permitting the use of a condensed tissue frame, 
may improve locoregional control by diminishing tumour cell regeneration between irradiation treatments (Thames et al, 1983). An early report of the MD Anderson Cancer Center suggested the potential benefits of using hyperfractionated radiotherapy that were confirmed in a later study (Barker et al, 1980; Liao et al, 2000). In a previous study, bifractionated irradiation was used after three cycles of chemotherapy and gave a very good rate of local control of $72 \%$ (Arriagada et al, 1990).

The timing of radiotherapy varied in the different studies: current practices delivered radiotherapy after chemotherapy and do not use it to obtain a rapid response rate.

In the literature, the role of surgery in IBC has been controversial. In the only prospective randomised study comparing surgery $v s$ radiotherapy following neoadjuvant chemotherapy in 57 patients with IBC, Mourali et al (1993) did not find any significant difference in DFS between the two groups. De Boer et al (2000) didn't find any difference in OS, DFS and disease-free relapse in a retrospective comparison between two groups of IBC patients: one treated by surgery and radiotherapy and the other by radiotherapy alone after neoadjuvant chemotherapy. But patients with complete clinical response were encouraged to have radiotherapy alone. In the trial of MD Anderson (Fleming et al, 1997; Ueno et al, 1997) in 178 patients, the authors found that patients who had response to induction chemotherapy benefited from the addition of mastectomy to chemotherapy and radiation. A recent retrospective analysis of 485 IBC patients has suggested that mastectomy in association with chemotherapy and radiotherapy seemed to enhance locoregional control with no impact on survival, but patients without mastectomy had more extensive disease at presentation. In our study with radio-chemotherapy, we achieved a high clinical control rate without mastectomy $(88 \%)$, which is confirmed by the long follow-up of 55.5 months with only six local recurrences in patients in complete clinical response (four with concomitant metastatic evolution) occurring in a median delay of 20 months.

Many different regimens of chemotherapy have been used, but anthracycline based chemotherapy remains the reference of systemic treatment (Chevallier et al, 1993; Mourali et al, 1993; Bauer et al, 1995).

In their retrospective study of 308 patients, Panades et al, 2005 found that a more intense chemotherapy regimen improved 10 years breast cancer specific survival. Other series have found no improvement on outcome with intensification (Therasse et al, 2003). In our protocol, after 24 patients we intensified the dose of induction chemotherapy as described, and no difference in local response was found before and after this intensification. On the other hand, we were unable to statistically assess the impact of chemotherapy dose on survival. Methotrexate was used after induction treatment for its good meningeal diffusion, but six brain or meningitis metastases occurred. Likewise, the role of maintenance chemotherapy must be elucidated.

The published survival data were obtained in historic trials or in retrospective studies based on single institution experience and have reported that at least one-third of patients treated with multi modality therapy are alive at 5 years. A recent report of the National Cancer Institute based on epidemiologic survey between 1988-1990 and 1997-1999 showed a poor median survival in IBC with 2.9 years (Hance et al, 2005). A larger retrospective trial reports the experience of the MD Anderson Cancer Center with a long follow-up of 7.4 years in 178 IBC patients treated with different multimodality protocols during a 20 -year period: the median survival was 37 months for all patients. Overall survival at 5 and 10 years was 40 and $33 \%$, respectively. Other studies reporting a long follow-up for IBC achieved an OS at 5 years from 34 to $75 \%$ (Liao et al, 2000; Harris et al, 2003; Baldini et al, 2004; Liauw et al, 2004), at 10 years range, respectively, from 13 to $47 \%$ (Low et al, 2004; Cariati et al, 2005) and a median OS between 25 to 62 months.

Many questions are not resolved in IBC treatment, such as the contribution of new drugs like Taxanes, the duration of chemotherapy, the best local treatment and the role of new molecular markers. However, the results obtained with initial dose dense chemotherapy and concomitant irradiation permit the proposal of this association as a useful therapeutic option.

\section{ACKNOWLEDGEMENTS}

We thank Sophie LEOBON and Nathalie PLANCHAT for their technical assistance.

\section{REFERENCES}

Anderson WF, Chu KC, Chang S (2003) Inflammatory breast carcinoma and non inflammatory locally advanced breast carcinoma: distinct clinicopathologic entities? J Clin Oncol 21: 2254-2259

Arriagada R, Mouriesse H, Spielmann M, Mezlini A, Oudinot P, le Chevalier T, Cuvier C, Fontaine F, Travagli JP, May-Levin F (1990) Alternating radiotherapy and chemotherapy in non-metastatic inflammatory breast cancer. Int J Radiat Oncol Biol Phys 19: 1207-1210

Baldini E, Gardin G, Evagelista G, Prochilot T, Collecchi P, Lionetto R (2004) Long-term results of combined-modality therapy for inflammatory breast carcinoma. Clin Breast Cancer 5: 358-363

Barker JL, Montague ED, Peters LJ (1980) Clinical experience with irradiation of inflammatory carcinoma of the breast with and without elective chemotherapy. Cancer 45: 625-629

Bauer RL, Bush E, Levine E, Edge SB (1995) Therapy for inflammatory breast cancer: impact of doxorubicin-based therapy. Ann Surg Oncol 2: 288-294

Cariati M, Bennett-Britton TM, Pinder SE, Purushotham AD (2005) 'Inflammatory' breast cancer. Surg Oncol 14: 133-143

Chang S, Parker SL, Pham T, Buzdar AU, Hurting SD (1998) Inflammatory breast carcinoma incidence and survival: the surveillance, epidemiology, and end results program of the national cancer institute 1975-1992. Cancer 15: 2366-2372

Chevallier B, Bastit P, Graic Y, Menard JF, Dauce JP, Julien JP, Clavier B, Kunlin A, D'Anjou J (1993) The center $\mathrm{H}$ Bequerel studies in inflammatory non metastatic breast cancer. Combined modality approach in 178 patients. $\mathrm{Br}$ J Cancer 67: 594-601

Cristofanilli M, Buzdar AU, Hortobagyi GN (2003) Update on the management of inflammatory breast cancer. Oncologist 8: 141-148

De Boer RH, Allum WH, Ebbs SR, Gui GP, Johnston SR, Sacks NP, Walsh G, Ashley S, Smith IE (2000) Multimodality therapy in inflammatory breast cancer: is there a place for surgery? Ann Oncol 11: $1147-1153$

Fleming RY, Asmar L, Buzdar AU, McNeese MD, Ames FC, Ross MI, Singletary SE (1997) Effectiveness of mastectomy by response to induction chemotherapy for control in inflammatory breast carcinoma. Ann Surg Oncol 4: 452-461

Haagensen CD (1971) Diseases of the Breast 2nd edn, Philadelphia: WB Saunders Company, pp 576-585

Hance KW, Anderson WF, Devesa SS, Young HA, Levine PH (2005) Trends in inflammatory breast carcinoma incidence and survival: the surveillance, epidemiology, and end results program at the national cancer institute. J Natl Cancer Inst 97: 966-975

Harris EE, Schultz D, Bertsch H, Fox K, Glick J, Solin LJ (2003) Ten-year outcome after combined modality therapy for inflammatory breast cancer. Int J Radiat Oncol Biol Phys 55: 1200-1208

Lerebours F, Bieche I, Lidereau R (2005) Update on inflammatory breast cancer. Breast Cancer Res 7: 52-58 
Liao Z, Strom EA, Buzdar AU, Singletary SE, Hunt K, Allen PK, McNeese MD (2000) Locoregional irradiation for inflammatory breast cancer: effectiveness of dose escalation in decreasing recurrence. Int J Radiot Oncol Biol Phys 47: $1191-1200$

Liauw SL, Benda RK, Morris CG, Mendenhall NP (2004) Inflammatory breast carcinoma: outcomes with trimodality therapy for non metastatic disease. Cancer 100: $920-928$

Low JA, Berman AW, Steinberg SM, Danforth DN, Lippman ME, Swain SM (2004) Long-term follow-up for locally advanced and inflammatory breast cancer patients treated with multimodality therapy. J Clin Oncol 22: $4067-4074$

Mourali N, Tabbane F, Muenz LR, Behi J, Ben Moussa F, Jaziri M, Levine PH (1993) Ten-year results utilizing chemotherapy as primary treatment in non metastatic, rapidly progressing breast cancer. Cancer Invest 11: $363-370$

Panades M, Olivotto IA, Speers CH, Shenkiert T, Olivotto TA, Weir L, Allan SJ (2005) Therapy of inflammatory breast cancer: impact of doxorubicinbased therapy. J Clin Oncol 23: $1941-1950$

Schafer P, Alberto P, Forni M, Obzadovic C, Pipard G, Krauer F (1987) Surgery as part of a combined modality approach for inflammatory breast carcinoma. Cancer 59: 1063 - 1067

Somlo G, Frankel P, Chow W, Leong L, Margolin K, Morgan Jr R, Shibata S, Chu P, Forman S, Lim D, Twardowski P, Weitzel J, Alvarnas J, Koqut N,
Schriber J, Fermin E, Yen Y, Damon L, Doroshow JH (2004) Prognostic indicators and survival in patients with stage IIIB inflammatory breast carcinoma after dose-intense chemotherapy. J Clin Oncol 22: $1839-1848$

Thames HD, Peters LJ, Withers HR (1983) Accelerated fractionation vs hyperfractionation: rationales for several treatments per day. Int J Radiat Oncol Biol Phys 9: 127-138

Therasse P, Mauriac L, Welnicka-Jaskiewicz M, Bruning P, Cufer T, Bonnefoi H, Tomiak E, Pritchard KI, Hamilton A, Piccart MJ, EORTC (2003) Final results of a randomized phase III trial comparing cyclophosphamide, epirubicin, and fluorouracil with a dose-intensified epirubicin and cyclophosphamide+filgrastim as neoadjuvant treatment in locally advanced breast cancer: an EORTC-NCIC-SAKK multicenter study. J Clin Oncol 21: $843-850$

Tubiana-Mathieu N, Lejeune C, Bonnier P, Genet D, Adjadj DJ, Berdah JF, Muracciole X, Delaby F, Clavère $\mathrm{P}$, Benyoub A, Roullet B, Rhein B, Juin P, Piana L (2001) Chemotherapy and concomitant irradiation in inflammatory breast cancer. Anticancer Res 21: $3061-3067$

Ueno NT, Buzdar AU, Singletary SE, Ames FC, McNeese MD, Holmes FA, Theriault RL, Strom EA, Wasaff BJ, Asmar L, Frye D, Hortobagyi GN (1997) Combined-modality treatment of inflammatory breast carcinoma: twenty years of experience at M. D. anderson cancer center. Cancer Chemother Pharmacol 40: 321 